\title{
DESIGN CONSIDERATIONS FOR A FIBER OPTIC COMMUNICATIONS NETWORK FOR POWER SYSTEMS
}

\author{
Harold Kirkham \\ Senior Member \\ Alan R. Johnston \\ Jet Propulsion Laboratory \\ California Institute of Technology \\ Pasadena, California
}

\author{
George D. Allen \\ Senior Member \\ American Electric Power \\ Service Corporation \\ Columbus, Ohio
}

$$
\text { AI05-79ET } 29372
$$

\begin{abstract}
The design of a fiber optic communication network for monitoring and control in power systems is discussed. It is shown that by appropriate choice of protocols, a fault-tolerant system can be built that operates in any arbitrary configuration.

Since the network is based on fiber optics, it can be made fast enough for substation monitoring and control. In this application, a relatively small number of cables is required to implement a high reliability system. The network can also be used for distribution automation. In this application the network is required to reach all parts of the power system, and the fiber cable itself becomes a significant fraction of the cost of communications. However, since many applications can be supported at once, the cost per function can be reasonable.
\end{abstract}

Keywords: Fiber optics, communications, substation automation, distribution automation, traffic study

\section{INTRODUCTION}

In this paper we will discuss a fiber optic communication system designed for monitoring and control of electric power systems. The power system requirements lead to a novel design: the assumptions usually made in designing generalpurpose computer communication systems do not apply. There are two particular differences. First, local area networks (LANs) assume any user is likely to require communication with any other with equal probability, whereas most control applications are hierarchical (master-slave), and peer-to-peer communication is rare. Second, LAN topologies are usually fixed in advance, and are rather simple

93 WM 029-9 PWRD A paper recommended and approved by the IEEE Power System Communication Committee of the IEEE Power Engineering Society for presentation. at the IEEE/PES 1993 Winter Meeting, Columbus, $O H$, L January 31 - February 5 1993. Manuscript submitted August 27 1992; made available for printing November 101992 . (loops or buses). In contrast, the configuration of a monitoring system may have to change as the power system evolves. Further, the system may have to continue to operate even if some fibers are damaged. The goal of our design effort was a communication system that could operate with any network topology, and would he reliable even if some tibers were damaged, or some terminals inoperative.

\section{DESIGN PHILOSOPHY}

We wanted to design a communication system that would meet all the worst-case requirements of a power system. This means that

- it could access as many locations as necessary to support monitoring or control functions,

- it could handle the highest data rate likely to be required by any foreseeable application,

- and it would continue to operate even if part of the network were damaged.

Essentially, we wanted a communication system that w.uld be the "phone company" for any and all automation functions. In this phone service mode, the application would not need to know how its messages were delivered, and the communication system would not need to know what the messages contained.

Consider first a communication system for use in the distribution system. The application imposes a requirement that the communication system reach everywhere on the power system. so that any monitoring or control function can be supported. Thus the topology of the network outside the substation is congruent with the distribution system. This means that each time there is a lateral in the distribution system. for example, there must be a spur in the communication system. A master node would be located at each disIribution substation. An example is shown in Figure 1.

In the Figure, each box represents a location on the power system where there is a need to perform control or monitoring. This becomes a node on the communication ststem. Normally, the distribution system is operated radially. There is a limited set of loops that are normally open, but that can be reconfigured to provide an alternative way of bringing power to any given location. The fiber cinn. of course, cross an open power switch. As a result. the tiher optic communication system is arranged not as a cont ventional ring, star or bus system, but as a series of interconnected loops, with an occasional spur. 


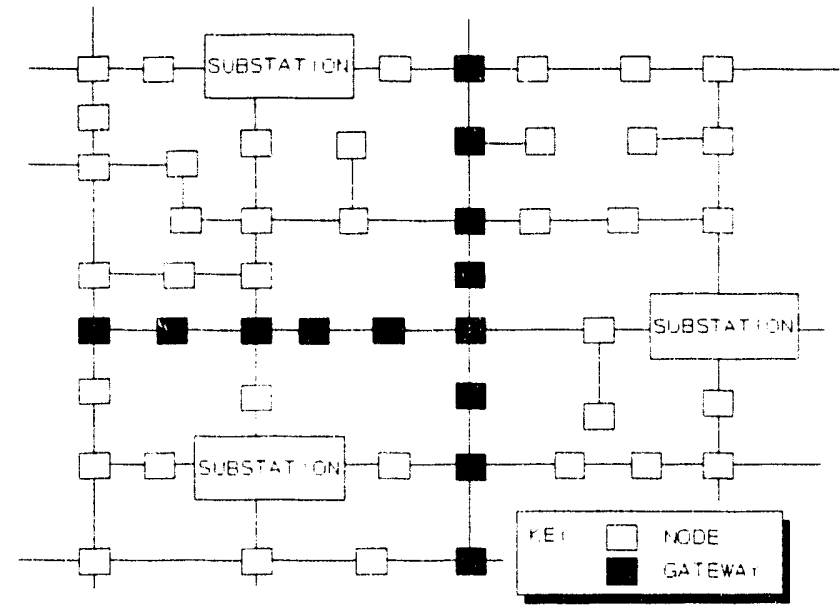

Figure 1. Representative topology of fiberbased communication system

The service territory of a substation defines an area containing slave nodes "owned" by the master. These nodes respond only to messages from their master. Some nodes are designated "gateways." A gateway is a node that is owned in the usual sense, but that can also respond to signals from a master in an adjacent area. We will see later that this can impact system reliability.

This is a somewhat unusual topology for a fiber optic network, in that there is a large number of interconnected loops with common legs, and many branch points. An important question is, How should the optical arrangement at the nodes or RTUs be implemented? The large number of points at which the fiber must be accessed for data acquisition or control means that ordinary optical taps cannot be used. Our solution is for each RTU tap point to include optical-electrical-optical conversion, that is, it functions as a repeater.

The same approach can be used for substation monitoring and control, except that the distances are shorter. In a substation, the location of the power apparatus (CTs, PTs, breakers) and the control building (which would house the master node) fixes the data acquisition geography. Fibers can be used to connect RTUs at these locations, and again the topology is unlikely to be simple. A substation automation scheme would differ topologically from a distribution automation scheme only in that spurs would be unlikely, for reasons of reliability.

This means that in both distribution system and substation applications the communication system is rather liberally sprinkled with repeaters. At the channel data rates typical of most fiber systems, the data blocks would occupy a rather small time. This implies that a communication approach that does not establish and maintain connections between nodes, but rather stores and forwards packets between them in a connectionless way might be efficient. A packet switching network with multiple rings and spurs represents an architecture whose possibilities and problems have not been discussed extensively in the literature.

A communications protocol that enables each master node to communicate reliably with all its RTUs has been developed and is being tested. It is described next.

\section{COMMUNICATION SYSTEM DESIGN}

\subsection{System Protocols}

The problems to be solved in a communications network include message routing, access to the medium, error detection and congestion control. These problems have been addressed in a number of ways by network designers. A step towards standardization of computer interconnections was taken when the Open Systems Interconnections model of the International Standards Organization was released [1].

The model splits the problem of computer communications into seven logical and physical layers. While few existing networks adhere strictly to this breakdown (and almost none attempt to define any layer higher than the network layer), we have found it advantageous to place our consideration of the communications problem firmly within this framework.

\subsection{Network Layer}

We begin this description of the protocols with the network layer because it is this layer that most differentiates our system from others. Perhaps even more than the other layers, we designed this layer for the kind of high-reliability low-cost communications needed in a power system.

The primary problems of the network layer are controlling the flow of data in the network, and routing within the network. It is instructive to see how these problems are solved in other networks. In a system such as Ethernet (ANSI/IEEE Standard 802.3-1985), routing is automatic because the hardware is based on parallel connections to a shared channel. All devices operate at the same speed. but there can be a problem when more than one station tries to transmit at the same time. (The event is called a collision).

In a communications system that has only one ring, routing is again automatic. Access can be controlled by a "token" passed from one station to the next. An example of this approach is ANSI/IEEE Standard 802.5-1985. The method may be explained as follows. When no node has any need to pass information, only a token circulates. When a node needs to communicate, it first removes the token from the communication line, thereby preventing other nodes from doing so. It then inserts its message. At the end of a message, another token is inserted, so that other nodes can access the ring.

Another standard protocol, IEEE 802.4 (Token bus), is a kind of hybrid. Implemented in fiber optics, it uses a star coupler so that all nodes are in parallel. Logically, it behaves like a ring.

None of these procedures adapt readily to the multiplering nature of our communications system. They do not solve the routing problem, nor can they easily prevent endless message circulation in a multiple-ring system.

For routing our network uses a flooding algorithm. This is a distributed solution. At all nodes, any message received is retransmitted on all outgoing lines. The message floods the entire system. By adopting such a strategy, a message inserted anywhere into the communications network shown in Figure 1 will eventually be broadcast to all units in the network. No message has any specific route; in fact all 
messages take all possible routes. This has two advantages: the shortest route is always taken, since all routes are always explored; and the transmission is very fault tolerant, since it does not depend on any particular path. The robust performance that this provides is important in many applications.

Of course, the message must be removed, too, to prevent endless circulation. In order to effect a distributed strategy to solve the circulation problem, every message contains a unique identifying number that can be stored in every node that it passes through. Each node decides whether or not the message will be repeated depending on whether the message has been seen before, or is addressed to the node in question. The overhead required to implement this is small. One or two bytes, added to the message, will suffice for message numbering.

The communications protocol resembles the working of the body's immune system. The first exposure to an invading organism causes it to learn (at the cell level) to recognize the organism as "non-self." On a second exposure, antibodies will be produced that kill the invader. In our communications network, the messages are the invading organism, and they wander throughout the network as far as available communications channels will allow. On first exposure to a message, our nodes store information that will allow them, on a second exposure, to recognize and kill the message. It is because of this similarity that one of our group proposed we call the system "AbNET," after the microbiologists" abbreviation "Ab," for antibody.

The method is quite efficient. The flooding algorithm means that all messages go down all channels. The antibody algorithm means that all links transmit the message exactly once. The combination means that changes to the network configuration, even during operation, can easily be accommodated.

\subsection{Data Link Layer}

The data link layer, underneath the network layer, is responsible for delimiting data fields, acknowledgement of receipt of data, and some error control, such as parity check. In most communications systems, receipt of information that passes the error check is acknowledged to the sending station. (The detection of an error will normally cause a request for retransmission.) In addition to this kind of function, the data link layer may cortain a flow control mechanism, to prevent problems when two devices of different speeds try to communicate .

Some simplifications are possible in our system. For example, the bit rate of all nodes can be fixed in advance, so flow control should not be needed. However, there seems to be little to be gained by retaining the conventional technique of message receipt acknowledgement. With a message 1000 bits long and a BER of $10^{-9}$, typical of fiberbased systems, the success probability in a normally operating link is $99.99990 \%$. In other words, only one message in a million would require retransmission or correction. It is inherently wasteful to devote any amount of effort to acknowledging successful reception of data. It seems likely that uncorrectable communication problems in a fiber optics network indicate a more severe problem than could be addressed by the data link layer.

By not employing a retransmission-request procedure, we can at least ensure that all traffic in our network is associated with the first-time sending of messages. No link will be made busy by nodes trying to overcome neighbor-toneighbor communications problems. In combination with the antibody algorithm, this helps guarantee that there will be no network congestion.

\subsection{Physical Layer: A Hybrid System}

The physical layer is the layer at which signals are exchanged. In our design, the physical layer is primarily based on fiber optics, but may be a hybrid (for reasons of economy) when used for distribution automation. Along the distribution feeders, or in the substation, multimode fiber is used. Transmitting optics are based on LEDs operating in the near infra-red. The bit rate (at least in our prutotypes) is $10 \mathrm{Mb} / \mathrm{s}$, because at speeds greater than about this figure, component costs rise sharply. Differential Manchester coding is used.

For substation automation, this is all that is required. In the distribution system, a fiber communication channel could be used all the way to the customers' homes. While this is technically feasible, cost considerations make a hybrid communication system worth considering. In particular, the use of fiber optics to the domestic customer's premises would mean access to the home, as well as modification of existing designs of load management equipment. We propose instead a hybrid system consisting of fiber optics on the distribution feeder and low-power high-frequency power line carrier communications on the secondary. The small size of the network to be covered (typically 3 or 4 houses) on the secondary greatly eases its communications requirements. Very low power, inexpensive modules operating at about $120 \mathrm{kHz}$ are widely available, and signalling speeds up to 1200 baud are possible. An example of this kind of system (actually UHF radio and DLC) is used in the NetComm project in California [2].

Low-data-rate functions such as demand-side management, can be handled with this kind of low-cost system, and there is no need to add wiring to the consumers premises.

Further, communication into the home can be compatible with other communication applications of the home wiring, for example the PLBus of the Electronic Industries Association (EIA) Home Automation Standard. An EIA PLBus interface at each distribution transformer would allow the utilities to accomplish far more control than simple management of one load. Not only could there be the capability of emergency load-shedding, but a solution to cold load pickup problems would be available.

\subsection{The Higher Layers}

In local area networks, it is generally assumed that each node is as likely as any other to originate a message. It is this assumption that leads to the need to solve the contention problem for access to the medium. Similar assumptions have generally been made the past in designing communi- 
cations systems for the distribution system. Because of data rate limitations, it has usually been necessary to limit communications traffic by having the RTUs make some intelligent decisions on their own. RTUs are often designed to operate with software that permits them to originate a transmission only if they detect some drastic change in the data they monitor. This approach is sometimes called Report-by-Exception.

Since the cause of such a change cannot be determined in advance, and might affect several RTUs simultaneously, some kind of medium access protocol must still be used. Collision detection has been used in some demonstrations of distribution automation, and is an automatic feature (indicated by a busy signal) of telephone-based communications. The problem with this solution is that the response to a collision is always a delay. Whenever widespread change occurs, the communications system becomes overloaded. Information transfer is subject to unpredictable delays when a quick response is most needed.

This is not the case with our system. Since the power system operating software is generally hierarchical, with centralized decision making and decentralized monitoring and control, we opted to use a centralized polling strategy at the transport layer. An RTU is thus a "slave," and can transmit only if so directed by the master node.

There are several options in our poling protocol. In one. the poll is directed to specific RTUs, and the response is returned along all lines going out from the RTU. This pure flooding approach provides maximum reliability. In a way this is a token-based system, but only the substation unit can originate a token, and that is addressed to a particular RTU.

In another option, the operating speed is increased, at the cost of a small decrease in the reliability. The poll is broadcast, and the response returned by each RTU only in the direction from which the poll arrived. The other output lines are used at the same time to repeat the poll. This option renders the network vulnerable to fiber failures that occur between the time of arrival of the poll and the transmission of the response. In view of the speed of the network, this is probably an acceptable risk.

Factors other than the polling approach used affect the speed with which data can be acquired. This is particularly true for distribution automation. It may be, for example. that a number of the RTUs in an area are simply taps into the fiber for the purpose of demand-side management. Data from nodes such as these will not be needed frequently, so they should be programmed to ignore a broadcast-type poll. With techniques like this, the time required for a scan of all nodes of even a large area can be made quite small.

\subsection{System Speed}

In a 1981 paper for a Panel Session on Automated Distrihution at the IEEE Conference on Overhead and Underground Transmission and Distribution, Carr [3] made the observation that no study comparing the information transfer needs of distribution automation with the capabilities of the various media seemed to have been published. Since, surprisingly, this is still largely the case, we have estimated the communication requirements of a typical $12-\mathrm{kV}$ line $12 \mathrm{~km}$ in length, as a reference model. A response time of $3 \mathrm{~s}$ is assumed for the remote terminal units (RTUs), representative of practice for SCADA-based systems. The following monitoring points are assumed: 1 station breaker (15 points), 3 line reclosers (33 points), 10 overhead distribution switches (110 points), 3 capacitor banks ( 21 points), 1 line regulator ( 9 points) and 10 single-phase line taps (30 points). The total number of points being monitored is thus 218 , from about 24 RTUs.

Using a compact format such as the IEEE Recommended Practice for Master/Remote Communications [4], it might take 242 bits for 16 points; therefore a little over 3 kbits are required to describe the feeder. Allowing 3 seconds for a scan, the bit rate is about $1000 \mathrm{bit} / \mathrm{s}$ per feeder. This is certainly modest by the standards of communication systems.

We feel it is more realistic to analyze a system that uses a general-purpose data representation. Assume that the data from one RTU alone occupies $3 \mathrm{~kb}$, and assume the message header (consisting of information identifying the message source, destination and number), to be $1 \mathrm{~kb}$. These assumptions increase the one-way average data rate on a feeder $10(4 \mathrm{k} \times 24 / 3)=32 \mathrm{~kb} / \mathrm{s}$. In a practical fiber optic system the traffic is bursty. Assuming a channel data rate of $10 \mathrm{Mb} / \mathrm{s}$, the approximately $100 \mathrm{~kb}$ from the example feeder could be communicated in about $10 \mathrm{~ms}$. (The slower protocol increases this time to about $60 \mathrm{~ms}$, still quite acceptable.) Allowing time for software execution might double this estimate. Note that the example of a single linear feeder provides a worst-case estimate of polling time. If the feeder is embedded in a network, it may be expected that shorter routes to some locations exist. The flooding algorithm would then yield a speed improvement. In the substation application a shorter route can be provided deliberately, at small additional cost.

\subsection{Reliability}

The network layer is inherently very tolerant of link or node failure. Even so, optical bypasses at the RTUs can further improve the resistance to node failure. With a bypass, if a node fails, or if the power to the node fails, the signal can still be passed through to downstream nodes.

While optical bypass components exist, they are not presently of sufficiently robust and economical design to be useful in this application. Work is presently underway to remedy this situation.

A feature of a highly fault-tolerant network is that failure of any particular connection may not be detected, and several failures may have occurred before there is a communication failure. There are a number of ways this drawback can be overcome; one of the more obvious ones is to have intermediate nodes update a routing table in the message header. Changes in the route taken by messages can then be logged. This approach increases packet size.

An alternative is to temporarily suspend the distributed flooding algorithm, and have the transport layer select a version of the network layer that is designed specifically to have each node identify its nearest neighbor. The remote nodes thus build their own table of neighbors, which they send to the master periodically. Because the message flow 
required for this task resembles the operation of Sonar, we have designated this a Ping. This approach takes time out of normal network operation.

Failure of a distribution substation unit can also be handled without overloading the communications channel. In the system diagram (Figure 1) some nodes were designated gateways. Since a gateway can pass signals from the area of one substation to another if required, communications can keep pace with the changing power system configuration. Should power be lost to a substation unit, control of its service area can be recovered, and assigned to neighboring substations.

\subsection{Voice Channel}

Finally, the system is designed to have a voice channel. This is not part of the packet switching network, and may seem at first to be rather wasteful-after all, it will rarely be used. However, it will be very convenient to have a voice channel during initial commissioning and subsequent maintenance of a distribution monitoring and control system. Such a channel will allow personnel installing or calibrating the equipment to talk directly with anyone else on the line, or with colleagues at the distribution substation.

Hardware is available that can multiplex voice and data onto the same channel. However, the requirements of the multiplexed voice channel would dominate the system design in our application. The bit rate required for the voice channel would exceed that of the data, and the timing requirements would be more critical.

It is not clear, however, that the voice channel should be added by multiplexing it cnto the fiber used for data acquisition and control. A separate fiber, or possibly two fibers, dedicated to voice communications, makes economical sense. The addition of a third and fourth fiber adds only about $10 \%$ to the cost of cable. A further advantage of a separate voice channel is that the data system and the voice system can be developed independently. It is also possible, of course, to build a "no-frills" version of the network, with no voice channel.

In each remote unit the voice fibers can either be terminated at an optical bypass as part of a special connector, or passed through low cost repeater equipment that could be tapped into as needed. The fibers need only be accessed at a few locations at any time, so they do not have to be managed as part of the general data acquisition and control system. A low-cost party-line system using pulsecode modulation (PCM) is perfectly adequate.

\section{COST COMPARISONS}

So far, we have not addressed cost. It may be that anticipation of high costs has prevented the widespread use of fiber optics in power system monitoring and control. We argue that fiber optics systems are presently available at economically attractive costs. While fiber optics are normally thought of as long-distance high-bandwidth systems, particularly because of their much-publicized applications in telephone trunk systems, the fact is that in a low-bandwidth short-distance system, fiber also makes economical sense.
In a fiber-based communications system, maximum use of resources is obtained by maximizing both the bandwidth and the distance between repeaters. Fewer fibers and fewer repeaters are then needed for a given amount of traffic.

For most telecommunications applications, the problem to be solved, then, has been one of increasing the data rate by improving the fibers and the opto-electronics. Lasers have to be developed that can insert more power into single-mode fibers; more sensitive receivers have to be developed.

However, for substation or distribution automation these arguments do not apply. We do not need to maximize the capability of the system. The number of repeaters is governed by the location of taps, fixed by the power system topology, not by cost considerations. The amount of traffic is rather modest, so easy-to-handle multimode fibers can be used. With multimode fiber and low data rates, high-power lasers and high-efficiency receivers will not be needed.

It follows that low-cost comnunications are possible. While we will argue below that an optical fiber system is needed because of technical and institutional advantages. we suggest first that the cost is actually so low it is comparable to other techniques.

For any communication network, capital costs as a function of distance can be written in the form:

$$
\begin{aligned}
& \text { Cost }=(\text { central unit cost }) \\
&+(\text { distance }) \times(\text { node cost }) \times(\text { number of } \\
&\text { nodes per unit length }) \\
&+(\text { distance }) \times(\text { channel cost per unit length })
\end{aligned}
$$

For substation use, we estimate that the cost of the central unit is comparable with the central unit of any other medium, such as pilot wires. The same is true of the remote nodes: the received signal is of such high quality that the hardware may actually be simpler and cheapur than for nodes using other media. Distances are so short that channel cost is probably negligible. In any case, the network is such that the length of fiber needed to implement a network is less than the length of pilot wire required to implement individual channels from master to nodes. The fiber network should thus be considered a cost-effective alternative to coris enumal media.

For distribution automation use, similar arguments can he made about node costs. The difference in system un between a fiber system and the conventional media is mamls the channel cost, which is zero for radio systems and 1$) \mid($. but not for telephone-based systems or fiber optics. WC have estimated that the fiber cable can be instailcd in .1 distribution circuit for about $\$ 3000$ per $\mathrm{km}$. (arr) $\mathrm{mL}$ charges at $10 \%$ on this amount of money over 31 sciar arc $\$ 26 /$ month. This distance is probably representatis spacing, and can be considered as part of the cost 11 c.k! node. The amount does not seem prohi'jitive.

It is important to note that the fiber optic system $124 \mathrm{mil}$ attractive as the number of applications increases $1 \mathrm{hm}$ because the incremental channel cost can be considcred th the zero once the first application is installed. For cx.muple suppose a feeder monitoring system is installed, whth a : wht (RTU) every $500 \mathrm{~m}$. Now, suppose load managemem in be added. The central unit and the channel are alrciut : 
place: all that is needed is the software, and the nodes for load management.

This option is not available with any of the conventional media normally considered for distribution automation. In the Guidelines for Evaluating Distribution Automation [5], it is observed that "A gap exists in that viable distribution communications systems for the fully automated distribution system are not available at the present time" (page 11-5). Some media are unsuited to access a large number of points, some support only one-way communications, some are capable of only low-rate signalling. Table 1 summarizes these deficiencies.

Table 1. Limitations of

Communications media

\begin{tabular}{ll}
\hline Medium & Limitation \\
\hline DLC & $\begin{array}{l}\text { Too slow ( } \approx 80 \mathrm{~b} / \mathrm{s} \text { max) } \\
\text { Needs intact power line } \\
\text { High error rate }\end{array}$ \\
& $\begin{array}{l}\text { One way } \\
\text { Radio (broadcast) }\end{array}$ \\
Radio (VHF and UHF) & $\begin{array}{l}\text { Limited coverage } \\
\text { Data rate only moderate } \\
\text { Multipath and shadowing }\end{array}$ \\
CATV & One way \\
Telephone (all kinds) & Access delays \\
& Limited number of points \\
\hline
\end{tabular}

There are institutional issues, too, that argue against several of the common media. For example, CATV and telephone channels involve some sharing of control over the medium, and some radio options require channel allocations from the FCC. In addition, a fiber-based approach is to be preferred because only software need be added, instead of new software and new communication hardware.

The more functions that are added, the greater the cost advantage of the fiber optic system. The conclusion is clear: a fiber optics communication system can be competitive in cost for node spacings that are realistic in many utilities.

\section{STATUS OF DEVELOPMENT}

A medium-sized network ( 24 nodes) has been simulated in software. The robust nature of the protocols is evident.

A laboratory-scale hardware demonstration of the system is presently under test. The system is based on PCs, interconnected by multimode fibers. Low-cost hardware, to convert electrical signals from the computer I/O into optical form, has been developed. The data rate is $10 \mathrm{Mb} / \mathrm{s}$.

Eventually, it is planned to test the system on a larger scale. An outdoor demonstration, using commercially developed prototype hardware, is in the planning stage.

\section{CONCLUSIONS}

This paper has briefly considered the application of fiber optics communications technology to the power system. A novel set of protocols has been designed for a network whose requirements are unlike general-purpose computer communication networks. The characteristics of the system are master/slave communications, moderate data rate, large number of access points, low cost and high reliability.

The combination of a flooding algorithm with antibodylike message removal and no handshake is very reliable and fault tolerant. It also guarantees the minimum transmission delay-the shortest route is always explored. Communications lines and $1 / O$ processors are never busy with unpredictable communications because of link or node problems. Each link carries messages once and only once, predictably.

A centralized polling algorithm ensures that communications traffic is completely under the control of the central unit-no RTU can access the network (other than as a repeater) unless so instructed by the central unit. Network performance can be centrally monitored. If necessary, the central unit can dynamically allocate priorities in the network. The high speed of the fiber network allows rapid sensing of the entire system, and use of error detection codes without impairing system performance. Communications can serve multiple functions, contributing to the economics.

The hybrid nature of the physical media allows low-cost implementation of low-performance applications such as load management. New functions involving customer-owned equipment (resetting clocks etc) can be added.

The strict organization according to the ISO OSI model, and the split between centralized and decentralized functions means that the lower layers can be adapted to other media (such as UHF radio for rural application) if advantageous. The split also segregates distribution automation from communications, ending vertical integration and possibly stimulating competition in both areas.

The performance of even a minimum fiber channel will permit the use of a flexible general-purpose data representation and at the same time retain an excess capacity of at least two orders of magnitude at no additional cost, a factor that may well change considerably the way in which system designers view the operation of the distribution system. Another way of putting this is that, even with a flexible data representation, system monitoring uses less than $1 \%$ of the channel capacity; the balance is available for future use.

It is concluded that, today, fiber optics is the appropriate choice of medium for monitoring and control in the power system hecause of its information bandwidth, dielectric nature, and probable installation cost.

Planners and designers and have worked in a bandwidthconstrained context up to the present time. Fiber optics will remove this constraint. There may well be new applications for the communications system that will take directions fitr from the original motivation. The history of similar advances in other technologies strongly suggests that other useful and unanticipated applications will in fact arise. Fiher hased communication systems of this kind may herald the heginning of a new era in utility operations.

\section{REFERENCES}

11) Zimmermann, H., (1980) "OSI Reference Model - the ISO Model of Architecture for Open Systems Intercon- 
nection" IEEE Transactions on Communications, Volume COM-28, No. 4, April, pp. 425-432.

[2] Holte, K.C., "Technology Requirements for a Competitive Electric Utility Market in the 21st Century" IEEE Power Engineering Review, Vol. 9, No. 7, pp. 18-22, July 1989.

[3] Carr, J., "Communication Requirements for Distribution Automation" Panel Session on Automated Distribution, IEEE Conference on Overhead and Underground Transmission and Distribution, Minneapolis $M N$, September 20-25. 1981).

[4] IEEE Recommended Practice for Master/Remote Communications, Working Paper WG 77.1, January 15, 1980. (See also Horstein, M. and Barnett, R., "Satellite Applications to Electric-Utility Communications Needs" JPL Publication 81-104, December 1, 1981 .

[5] Bunch, J., Guidelines for Evaluating Distribution Automation (EPRI EL-3728, Research Project 2021-1, Final Report, November 1984)

\section{ACKNOWLEDGEMENTS}

The authors would like to acknowledge the contributions of Shannon Jackson in the design and testing of network prototype hardware, and Heather Friend in writing simulation software and in the documentation.

Prepared by the Jet Propulsion Laboratory, California Institute of Technology, for the U.S. Department of Energy, Office of Energy Management Systems, Utility Systems Division, through an agreement with the National Aeronautics and Space Administration.

This report was prepared as an account of work sponsored by an agency of the United States Government. Neither the United States Government nor any agency thereof, nor any of their employees, makes any warranty, express or implied, or assumes any legal liability or responsibility for the accuracy, completeness, or usefulness of any information, product, or process disclosed, or represents that its use would not infringe privately owned rights.

\section{AUTHORS' BIOGRAPHIES}

Harold Kirkham (SM) was born in Birmingham, England in 1943. He received a B.Sc. (Hons) in 1966 and an M.Sc. in 1967 from the University of Aston in Birmingham. In 1973 he received a Ph.D. from Drexel University in Philadelphia.

In Philadelphia, he worked at the AC/DC Research Project of the Edison Electric Institute, and continued an interest in the topic of combined AC/DC systems into his Ph.D. work. From 1973 until 1979 he was with American Electric Power, responsi- ble for the data acquisition system at their UHV station in Indiana. In 1979 Dr. Kirkham joined the Communications and Control for Electric Power Systems project at the Jet Propulsion Laboratory in Pasadena, CA. Since 1984 he has been Project Manager.

Dr. Kirkham's current research interests are largely associated with power system applications of fiber optics. $\mathrm{He}$ is a member of the Fiber Optics Subcommittee of the Communications Committee of PES, and Chairman of a Working Group on Fiber Sensors.

Alan R. Johnston was born in Long Beach, California in 1931. He received the B.S. in Physics in 1952 and the Ph.D. in Physics in 1956. both from the California Institute of Technology.

Aside from a brief period as a Visiting Professor at Chalmers Technical University in Göteborg, Sweden, Dr. Johnston has been at the Jet Propulsion Laboratory, California Institute of Technology since 1956. He has worked on inertial guidance systems, electro- and ferro-optic materials, and proximity sensing devices for robotics. He initiated a fiber optics R\&D program at JPL, aimed at NASA applications. (An orbital fiber optics experiment was launched in April 1984.)

Dr. Johnston's current research interests are fiber sensing, optical computing and fiber networking as well as optical interconnection techniques. Dr. Johnston is a member of the American Physical Society, the Optical Society of America and Sigma $\mathrm{Xi}$.

George D. Allen (SM) was born in Jackson, MS in 1938. He received the B.S. in Electrical Engineering from Loyola University, Los Angeles in 1962, and the M.S. in Electrical Engineering from the University of Southern California in 1967.

After gaining his BSEE, he was employed at the Los Angeles Department of Water and Power as an engineer in the Relay Protection Department. From 1972 to 1978 he was Chief Engineer. Underground Switchgear. with A.B. Chance in Centralia, MO. From 1978 to 1980. he was Engineering Manager, Protection Products, at McGraw Edison in Olean, NY. He has been a Principal Engineer in the Distribution Section of American Electric Power Service Corporation since 1980.

Mr. Allen is a Senior Member of IEEE, and a licensed Professional Engineer in California and Ohio. He hals published extensively in IEEE and the American Power Conference, and holds 2 U.S. patents. 


\section{DISCLAIMER}

This report was prepared as an account of work sponsored by an agency of the United States Government. Neither the United States Government nor any agency thereof, nor any of their employees, makes any warranty, express or implied, or assumes any legal liability or responsibility for the accuracy, completeness, or usefulness of any information, apparatus, product, or process disclosed, or represents that its use would not infringe privately owned rights. Reference herein to any specific commercial product, process, or service by trade name, trademark, manufacturer, or otherwise does not necessarily constitute or imply its endorsement, recommendation, or favoring by the United States Government or any agency thereof. The views and opinions of authors expressed herein do not necessarily state or reflect those of the United States Government or any agency thereof. 

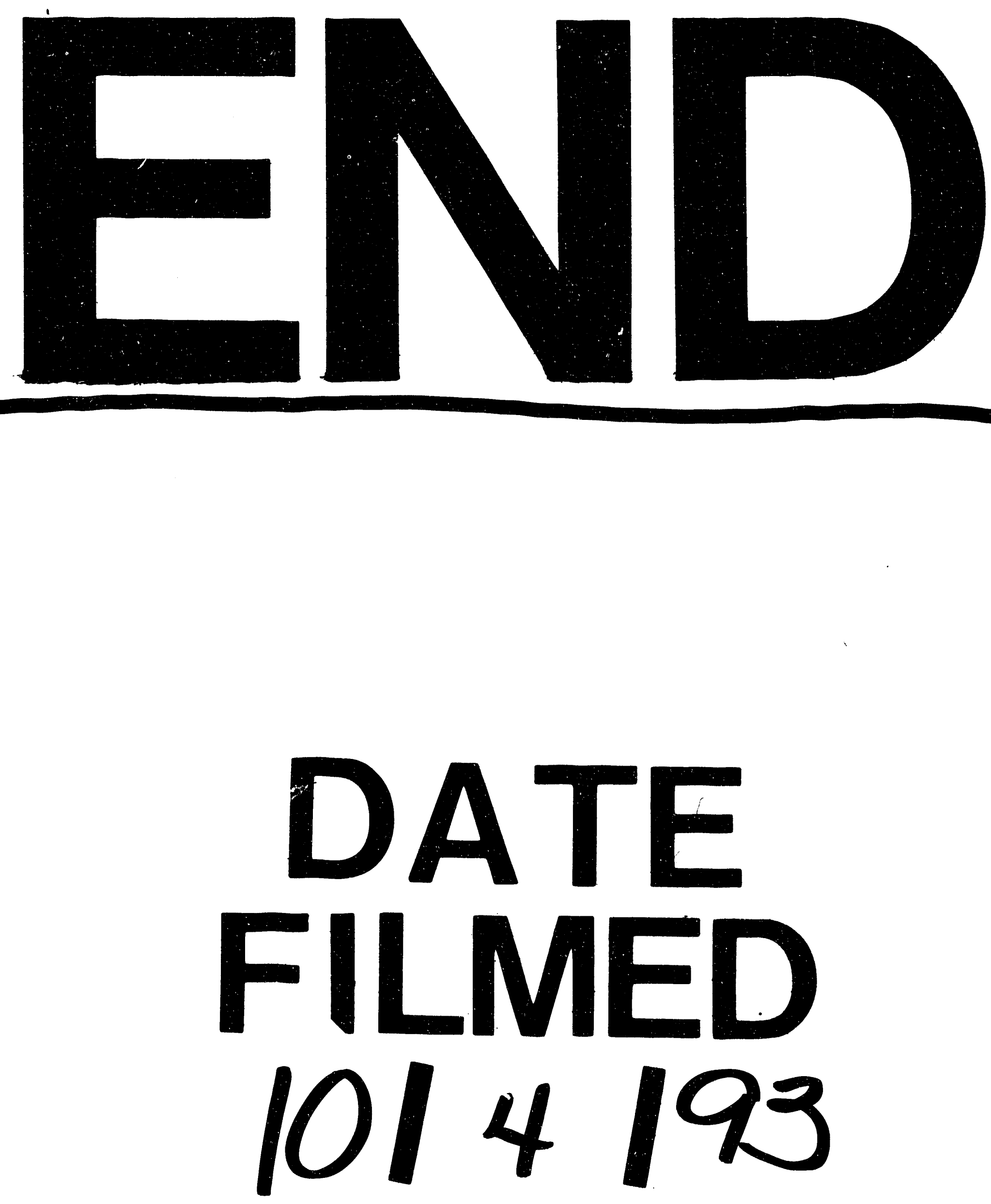
